\title{
BMJ Global Health Facilitating access to surgical care through a decentralised case-finding strategy: experience in Madagascar
}

\author{
Michelle C White, ${ }^{1,2,3}$ Mirjam Hamer, ${ }^{3,4}$ Jasmin Biddell, ${ }^{3,5}$ Nathan Claus, ${ }^{2,3}$ \\ Kirsten Randall, ${ }^{2,3}$ Dennis Alcorn, ${ }^{2}$ Gary Parker, ${ }^{2,3}$ Mark G Shrime ${ }^{6,7}$
}

To cite: White MC, Hamer M, Biddell J, et al. Facilitating access to surgical care through a decentralised case-finding strategy: experience in Madagascar. BMJ Glob Health 2017;2:e000427. doi:10.1136/ bmjgh-2017-000427

Handling editor Seye Abimbola

- Additional material is published online only. To view please visit the journal online (http://dx.doi.org/10.1136/ bmjgh-2017-000427).

Received 29 May 2017 Revised 26 August 2017 Accepted 31 August 2017

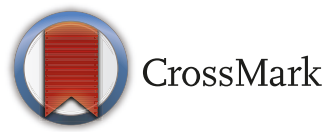

For numbered affiliations see end of article.

Correspondence to Dr Michelle C White; doctormcw@gmail.com

\section{ABSTRACT}

Over two-thirds of the world's population lack access to surgical care. Non-governmental organisation's providing free surgeries may overcome financial barriers, but other barriers to care still exist. This analysis paper discusses two different case-finding strategies in Madagascar that aimed to increase the proportion of poor patients, women and those for whom multiple barriers to care exist.From October 2014 to June 2015, we used a centralised selection strategy, aiming to find $70 \%$ of patients from the port city, Toamasina, and 30\% from the national capital and two remote cities. From August 2015 to June 2016, a decentralised strategy was used aiming to find $30 \%$ of patients from Toamasina and $70 \%$ from 11 remote locations, including the capital. Demographic information and self-reported barriers to care were collected. Wealth quintile was calculated for each patient using a combination of participant responses to asset-related and demographic questions, and publicly available data. A total of 2971 patients were assessed. The change from centralised to decentralised selection resulted in significantly poorer patients undergoing surgery. All reported barriers to prior care, except for lack of transportation, were significantly more likely to be identified in the decentralised group. Patients who identified multiple barriers to prior surgical care were less likely to be from the richest quintile $(p=0.037)$ and more likely to be in the decentralised group $(p=0.046)$. Our country-specific analysis shows that decentralised patient selection strategies may be used to overcome barriers to care and allow patients in greatest need to access surgical care.

\section{THE NEED}

Globally, 5 billion people lack access to safe, affordable and timely surgical care, ${ }^{1}$ and an estimated 143 million more procedures are needed each year to meet that need. ${ }^{2}$ Challenges to accessing surgical care are multifactorial and involve complex interactions between cultural, geographical, social and economic factors. The cost of surgery in low-income and middle-income countries (LMICs) is near prohibitive, with 81 million people facing catastrophic expenditure due to the costs of surgery per year. ${ }^{34}$

\section{Key questions}

What is already known about this topic?

- Globally 5 billion people lack access to safe, affordable and timely surgery.

- Barriers to surgical access involve complex interactions between cultural, geographical, social and economic factors.

What are the new findings?

- A decentralised patient selection strategy that actively targets poor and remote areas can increase access to patients in the lowest wealth quintiles.

- This strategy can increase access to patients who have previously experienced multiple barriers to care.

Recommendations for policy

- Active case-finding in the poorest communities, in collaboration with the community and its religious leaders, may work to overcome fear and mistrust, leading to an increase in access to surgery.

- Non-governmental organisations should consider case-finding by travelling to patient's rural locations, rather than expecting the patient to travel to access care.

The direct medical cost of the surgery itself is often not the largest portion of a patient's financial burden ${ }^{3}$ : patients' travel costs are the most commonly reported barrier to accessing cleft lip and palate surgery in sub-Saharan Africa, ${ }^{5}$ and gender disparities in access are also known to exist. ${ }^{6-10}$ Other documented barriers to care in LMICs include a lack of available surgeons, inadequate facilities, poor roads and low levels of education and health literacy. ${ }^{6-17}$ These barriers fall heaviest on the poor and those living in remote areas.

Non-governmental organisations (NGOs) play a significant role in providing surgical care in LMICs. In some countries, over $50 \%$ of the total surgical care is provided by NGOs. ${ }^{18}$ Lack of surgeon availability, inadequate facilities and surgical costs may be overcome by NGOs offering free surgical care, but 


\section{MADAGASCAR}

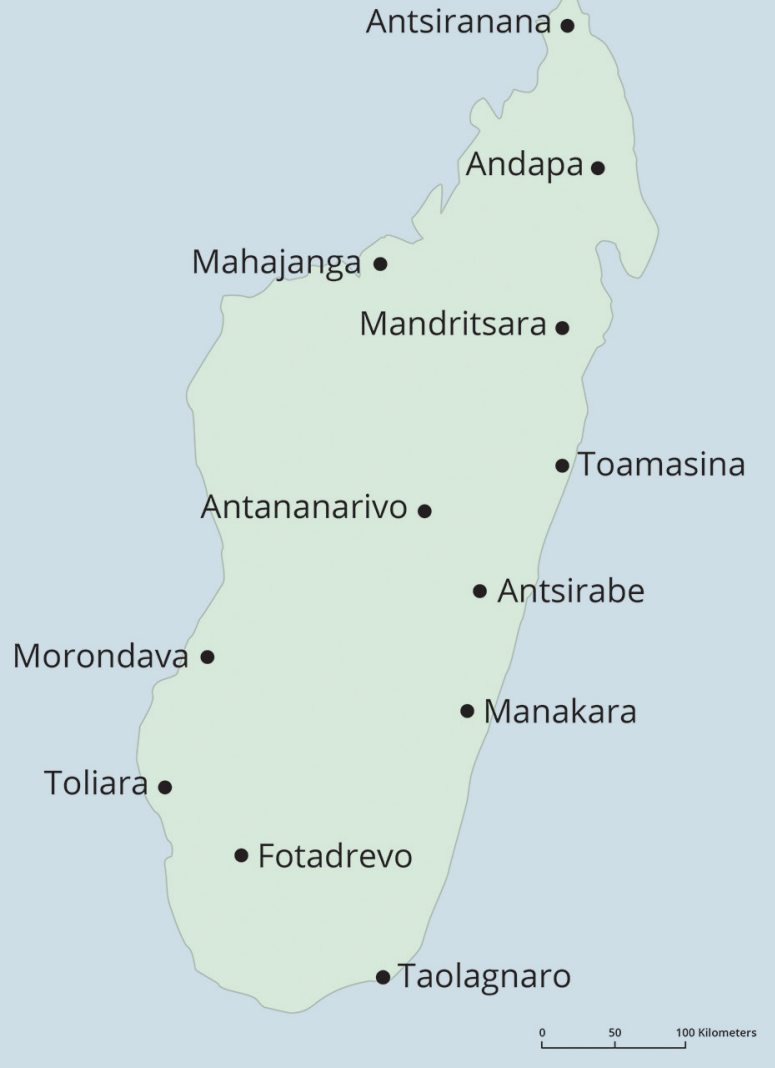

Figure 1 Map of Madagascar showing location of cities used in centralised and decentralised selection strategies.

other barriers still exist, such as distance, transport costs and health literacy. Deliberate patient selection strategies aimed to target the financially poor, those living in remote areas and to overcome gender disparities are needed, but it is unclear whether such strategies are sufficient to overcome these barriers.

Mercy Ships is a surgical NGO that operates the world's largest civilian hospital ship, the Africa Mercy. The Africa Mercy visits coastal sub-Saharan African countries at the invitation of the head of state, typically spending 10 months in one country docked in the major port city. Working closely with the Ministry of Health free surgeries, training and quality improvement initiatives are provided. The ship has 84 beds and 5 operating rooms providing a range of elective maxillofacial, plastics, general, gynaecological and orthopaedic procedures. Typical surgeries include cleft lip and palate surgery including pharyngoplasty; excision of soft tissue and bony tumours of the head and neck including those requiring mandibulectomy and maxillectomy; reconstructive surgery after noma or after burns to the head, neck and limbs and hands; excision of large soft tissue tumours such as neurofibroma; goitre surgery, obstetric fistula and hernia repair; and correction of neglected clubfoot, valgus and varus deformities of the lower limbs. Outpatient and rehabilitation services are provided during the 10-month period. Any patients needing further care after Mercy Ships departure are handed over to the care of the local hospitals usually to doctors who have participated in the Mercy Ships training programmes and a Memorandum of Understanding for costs arranged with the hospital director. Data on patient's socioeconomic status and self-reported barriers to surgical care are routinely collected, and Mercy Ships has previously examined the relationship between poverty, barriers to surgical care and health outcomes in the Republic of Congo. ${ }^{14}$

For two consecutive field services, from October 2014 to June 2015 and from August 2015 to June 2016, Mercy Ships was based in Madagascar, a low-income country off the east coast of Africa. Madagascar has a population of 24 million, a surgical workforce density of 0.78 providers per 100000 population, annual surgical volume of 135-191 procedures per 100000 population and a perioperative mortality rate of $2.5 \%-3.3 \% .^{19}$ Only $20 \%$ of the population can access surgical services within 2 hours, and up to $95 \%$ would face financial ruin if they required surgery. ${ }^{19}$ Most hospitals lack reliable electricity and oxygen supplies, basic monitoring required for safe anaesthesia and paediatric-appropriate equipment for surgery and anaesthesia. ${ }^{20}$

This analysis paper describes how Mercy Ships changed from a centralised to a decentralised patient selection strategy with the aim of recruiting a greater proportion of underserved patients. We report the impact of our changes with respect to patient's wealth index and self-reported barriers to care. Recommendations to other NGOs, policymakers and funders are given with respect to targeting patients in greatest need.

\section{THE PATIENT SELECTION PROGRAMME Mercy Ships' patient selection process} Initial assessment and design

The patient selection programme begins before the ship's arrival. Initial assessments are made to design a surgical schedule that meets host country needs. Assessments consist of interviews with key government personnel; representatives from the WHO, aid agencies and other NGOs, local hospital staff and community leaders. The assessment informs a collaborative decision-making process between Mercy Ships and the Ministry of Health concerning surgical programme design and consequent patient selection.

Historically, $70 \%-80 \%$ of Mercy Ships patients are selected at the beginning of a field service at a field site in the major port or capital city. Potential patients are expected to travel to this city to be seen. The remaining $20 \%-30 \%$ of the surgical capacity is reserved for patients travelling to two or three smaller screening events in remote areas identified by the Ministry of Health. Billboard, radio and television advertisements detail dates and locations of selection events and the types of 


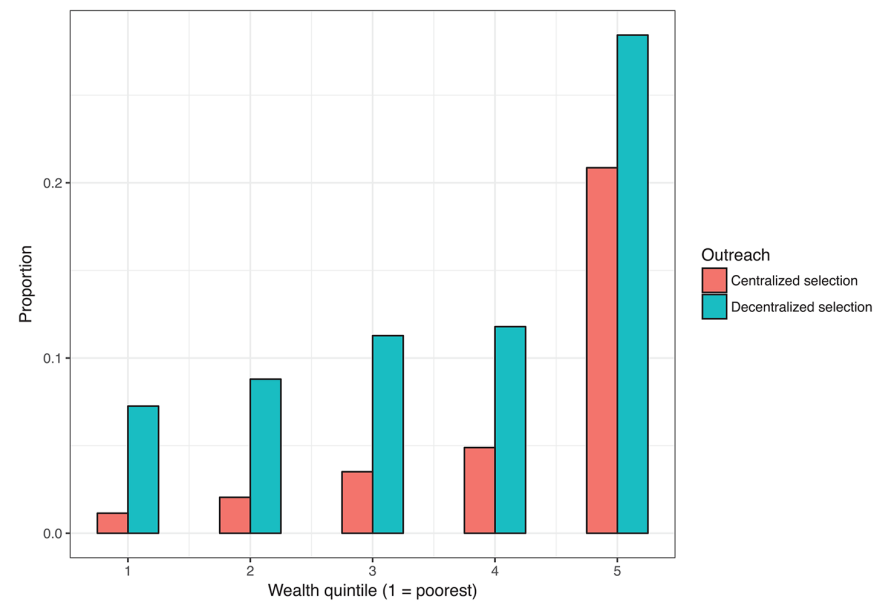

Figure 2 Proportions of patients defined by wealth quintile.

conditions treated. Local hospital contacts, community leaders and other NGOs also help spread the word.

\section{Change in selection strategy}

In Madagascar, the Africa Mercy was docked in the eastern port city, Toamasina, which is 8 hours by road from the capital city, Antananarivo. In the first field service (October 2014-June 2015), Mercy Ships used a centralised selection strategy, aiming to find $70 \%$ of patients from Toamasina and 30\% from the capital (Antananarivo) and two remote cities (Mahajanga and Toliara). From August 2015 to June 2016, a decentralised selection strategy was used, aiming to find only $30 \%$ of patients from Toamasina and $70 \%$ from the capital and 10 remote locations. For the decentralised screening strategy, the 10 remote sites were identified in collaboration with the Ministry of Health as sufficient to cover the majority of the country's population: Tolagnaro, Manakara, Mahajanga, Antsiranana, Mandritsara, Andapa, Toliara, Fortadrevo, Antsirabe and Morondava (see figure 1). In general, these 10 key cities were at least 1 day's travel from the capital city and at least 2 days' travel from Toamasina. Patients still had to reach the key city but, thereafter, surgery, patient transport, accommodation and food were provided free of charge. All cities remote from the port city were visited at least twice: once to perform an initial assessment including crowd security and plan the selection process with the Regional Minister of Health, local hospital leadership, community leaders, police and media and later to undertake the patient selection process.

The decentralisation strategy was initiated at the request of the Ministry of Health who wanted the rural populations to have equal opportunity to access Mercy Ships' specialised surgical services.

\section{Patient selection process}

The patient selection team consists of five nurses with experience working with Mercy Ships. These nurses organise the patient selection process that occurs in two phases: (1) nurse-led field selection (port/capital city and remote locations) and (2) surgeon-led dockside screening.

\section{Field selection}

Potential patients arriving at the selection venue and are rapidly assessed in three phases. Phase one is a rapid (1-2 min) assessment. Patients who do not fall within the spectrum of diseases for which Mercy Ships undertakes surgery are escorted away, while potential surgical candidates pass to phase two. Phase two consists of a more detailed assessment of the patient's presenting condition and general health. Suitable patients have their contact details recorded and pass to phase three where they receive a date for dock-side surgeon screening and details of transportation arrangements provided by Mercy Ships to get to the ship. Field selection sizes vary from 200 to 900 patients in phase one, with from 24 to 144 patients completing phase three, which represents $2 \%-4 \%$ of those presenting for selection. Despite billboard, radio and television announcements detailing the types of conditions treated and spending prior time educating local community and medical teams, many people present with conditions Mercy Ships cannot treat. These patients often report that they had been told Mercy Ships would unlikely to be able to help, but they 'just wanted to check and hear for themselves'.

\section{Dock-side surgeon screening}

All Mercy Ships surgeons screen their own patients from the predetermined group brought to the dockside by the selection team. This usually occurs the day after the surgeon's arrival. After surgical review, patients accepted for surgery are usually operated on within the next 2 weeks, since visiting surgeons usually rotate every 2 weeks. Dock-side surgeon screenings occur approximately every $2-3$ weeks for each specialty. The number of patients attending depends on the specialty and the number of surgeons performing the assessments but ranges from 20 to 60 patients.

The patient selection team nurses organise and assist surgeons at dock-side screening, which helps the nurses more accurately assess patients at remote field screenings. Over $85 \%$ of patients brought to dock-side screenings go on to receive surgery.

\section{THE EVALUATION \\ Study participants}

We evaluated all patients admitted for surgery on board the ${ }^{17}$ Africa Mercy between October 2014 and June 2016. Demographic information and self-reported barriers to care are routinely collected on all Mercy Ships patients at the time of their admission, ${ }^{14}$ via a questionnaire administered by trained translators fluent in English, French, Malagasy and local dialects. All patients are informed that treatment is free of charge regardless of their questionnaire responses. A copy of the demographic questionnaire is included in the online appendix file. 


\begin{tabular}{|c|c|c|c|c|}
\hline Coefficients: & Estimate & SE & z Value & $\operatorname{Pr}(>|z|)$ \\
\hline (Intercept) & -1.848 & 0.200 & -9.248 & $<0.001^{\star \star}$ \\
\hline Quintile: poor & 0.392 & 0.252 & 1.553 & 0.120 \\
\hline Quintile: middle & 0.680 & 0.234 & 2.910 & $0.004^{*}$ \\
\hline Quintile: rich & 0.968 & 0.227 & 4.270 & $<0.001^{* \star}$ \\
\hline Quintile: richest & 1.538 & 0.208 & 7.400 & $<0.001^{\star \star}$ \\
\hline
\end{tabular}

Significance: ${ }^{\star} p<0.01 ;{ }^{* *} p<0.001$.

A total of 2971 patients were assessed, 1156 in in the centralised selection group and 1815 in the decentralised selection group. Survey responses were insufficient to assign a wealth quintile for 333 and 102 patients in the centralised and peripheral selection groups, respectively, leaving 2536 patients for final evaluation.

\section{Derivation of wealth quintile}

Wealth index calculation, using a combination of participant responses to asset-related and demographic questions and publicly available country-specific data from the Demographic and Health Survey (DHS) programme, ${ }^{21}$ has previously been described. ${ }^{14}$ Briefly, DHS data are used to derive weights for each asset-related variable as it relates to wealth index; these weights are used to create a probabilistic microsimulation to assign a wealth index for individual Mercy Ships surgical patients.

\section{Ascertainment of barriers to care}

As part of the demographic questionnaire, patients are asked if they have had previous surgery for the presenting condition. If they respond in the negative, they are asked, 'what has prevented you from having your disease treated so far?'. Possible responses, of which respondents can select more than one, are: 'Treatment would have been too expensive', 'No surgeon was available', 'I was concerned about the quality of care', 'I did not have transportation/could not get to treatment', 'The distance to care was too far', 'I was needed at work', 'A spouse or family member would not allow access to care', 'I did not think I needed care/treatment'. Patients were also allowed to give other reasons.

\section{Statistical analysis}

The primary outcome measure was the difference in proportion of patients in the lowest wealth quintiles between the centralised and peripheral selection strategies. Secondary outcomes were the differences in barriers to surgical care, gender and age between the centralised and peripheral selection strategies.

For categorical variables, logistic regressions were performed to predict the probability of being seen with a peripheral selection strategy, as compared with a centralised selection strategy. For binary variables, Z-tests were performed.
Table 2 Barriers to care reported by participants in the two selection groups

\begin{tabular}{|c|c|c|}
\hline Barrier & $\begin{array}{l}\text { Centralised } \\
\text { selection }\end{array}$ & $\begin{array}{l}\text { Decentralised } \\
\text { selection }\end{array}$ \\
\hline No barrier identified & 377 & 107 \\
\hline $\begin{array}{l}\text { Treatment would have been } \\
\text { too expensive }\end{array}$ & 393 & 769 \\
\hline No surgeon was available & 30 & 124 \\
\hline $\begin{array}{l}\text { I was concerned about the } \\
\text { quality of care }\end{array}$ & 8 & 20 \\
\hline $\begin{array}{l}\text { I did not have transportation/ } \\
\text { could not get to treatment }\end{array}$ & 0 & 2 \\
\hline $\begin{array}{l}\text { The distance to care was too } \\
\text { far }\end{array}$ & 9 & 11 \\
\hline I was needed at work & 1 & 3 \\
\hline $\begin{array}{l}\text { A spouse or family member } \\
\text { would not allow access to } \\
\text { care }\end{array}$ & 10 & 12 \\
\hline $\begin{array}{l}\text { I did not think I needed care/ } \\
\text { treatment }\end{array}$ & 67 & 96 \\
\hline Multiple barriers identified & 25 & 87 \\
\hline Other barrier & 56 & 86 \\
\hline
\end{tabular}

\section{Ethical considerations}

Mercy Ships Institutional Review Board approved the study. The Institutional Review Board at the Massachusetts Eye and Ear Infirmary, where only deidentified data were analysed, deemed this study exempt.

\section{THE IMPACT}

The change in selection strategy from centralised to decentralised selection resulted in proportionally more patients in the lower wealth quintiles undergoing surgery. Figure 2 shows the proportion of patients in the five quintiles for the two selection methods. Patients in the richest, rich and middle quintiles were 4.6, 2.6 and 2.0 times, respectively, more likely to have been selected in the centralised selection strategy (table 1).

Table 3 Association between identification of multiple barriers to care and field service, age, sex and wealth

\begin{tabular}{lcccc}
\hline Coefficients: & Estimate & SE & z Value & $\operatorname{Pr}(>|z|)$ \\
\hline (Intercept) & -2.540 & 0.383 & -6.633 & $<0.001^{* * *}$ \\
Peripheral group & 0.479 & 0.240 & 1.995 & $0.046^{*}$ \\
Age & -0.006 & 0.0055 & -1.173 & 0.241 \\
Female & 0.115 & 0.205 & 0.562 & 0.574 \\
Quintile: poor & -0.213 & 0.369 & -0.577 & 0.564 \\
Quintile: middle & -0.389 & 0.357 & -1.089 & 0.276 \\
Quintile: rich & -0.461 & 0.358 & -1.289 & 0.198 \\
Quintile: richest & -0.697 & 0.323 & -2.16 & $0.031^{*}$ \\
\hline
\end{tabular}

Significance: ${ }^{*} p<0.05 ;{ }^{* *} p<0.01 ;{ }^{* *} p<0.001$. 
Patients ranged in age from 3 months to 73 years; the centralised group had a median age of 24 years (IQR: 7-42 years), and decentralised group had a median age of 21 years (IQR: 6-39 years). Patients in the decentralised group were younger than those in the centralised group, although this difference did not quite achieve statistical significance $(p=0.056)$. There were significantly more females than males ( $52 \%$ vs $47.4 \%, \mathrm{p}=0.022$ ) in the decentralised group. However, in collaboration with the government of Madagascar, we increased the numbers of obstetric fistula surgeries during the second field service and when this increase is controlled for, the effect of gender disappears.

The type of surgery and number of patients per specialty was consistent across the two field services with two exceptions. Due to a later than usual start to the first field service (October instead of August), the orthopaedic programme was reduced in the first field service (centralised group), and in the second field service (decentralised group), obstetric fistula surgery was increased at the request of the government. Average numbers of patients per specialty per month were maxillofacial: 39 and 41, plastic surgery: 11 and 10, general surgery: 40 and 45, orthopaedic surgery: 6 and 10 and obstetric fistula: 1 and 4 for the centralised and decentralised groups, respectively.

All reported barriers to prior care, except for lack of transportation $(\mathrm{p}=0.95)$, were statistically significantly more likely to have been identified by patients in the decentralised group compared with centralised group. More patients in the centralised selection group compared with the decentralised group reported no barriers to accessing care $(38.6 \%$ vs $8.1 \%, \mathrm{p}<0.001)$ (table 2$)$.

Patients who identified multiple barriers to prior surgical care were more likely to have presented in the decentralised group $(p=0.046)$ and less likely to be in the richest quintile $(p=0.037)$. Age and gender were not predictive of facing multiple barriers to care (table 3 ).

\section{OVERCOMING BARRIERS TO SURGICAL ACCESS FOR THE MOST UNDERSERVED}

This data-driven analysis demonstrates that a decentralised patient selection strategy that actively targets poor and remote areas can increase access to the most underserved, especially the poor and patients facing multiple barriers to care.

There are various models for expanding access to surgical care that include task-shifting, ${ }^{22}$ use of mobile platforms ${ }^{23}$ and impact of government policy, ${ }^{24}$ but little is known regarding case-finding strategy. We sequentially evaluated two selection strategies: a centralised selection strategy that focused on the larger population centres in Madagascar and a decentralised strategy that deliberately targeted remote areas of Madagascar, where access to surgical care was limited. The decentralised strategy aimed to gain widespread coverage of the whole country so that surgery was available to as many people as possible. Given that travel costs are the single most common barrier to accessing free cleft lip and palate surgery in sub-Saharan Africa, ${ }^{5}$ our findings suggest that NGOs should consider case-finding by travelling to patient's rural locations, rather than expecting the patient to bear the brunt of transport costs. This will require a change in strategy and higher upfront costs for many NGOs, but the return on this investment is the ability to reach a greater proportion of the rural population. However, in the poorest of countries, even richer urban patients may lack access to surgical care due to lack of trained surgeons, so a decentralised case finding strategy may just shift the population treated.

Fear of surgery and mistrust of service providers are also known barriers to care, ${ }^{11} 13151725$ and women are more than three times as likely as men to experience such fears. ${ }^{16}$ The results presented here suggest that active case-finding in the poorest communities, in collaboration with the community and its religious leaders, may work to overcome some of this fear and mistrust, leading to an increase in access to surgery by parent's willingness to present children for surgery. As the number of surgical NGOs grows, collaboration between NGOs and Ministries of Health is also likely to be important. Collaboration to avoid duplication of services, share patient details and facilities (eg, CT scanning) and provision of ongoing care are also likely to be important in overcoming barriers to care.

That patients in the decentralised group did not more readily identify lack of transportation is surprising given the remoteness of this population. A possible explanation for this may be that in this context due to the overwhelming barriers being surgical cost and lack of surgeons, the issue of transport was perceived as irrelevant unless the other barriers were overcome.

This analysis has a number of limitations. The barriersto-care questionnaire retrospectively asked patients if they had previously experienced barriers to care and so is subject to recall bias. There is no recent DHS programme data available for Madagascar, so we had to create a simulation model to allow weights to be given to the demographic and asset-related questionnaire that could have introduced errors. However, when the simulation model was applied to the original DHS data, the correlation between predicted wealth and actual wealth was $91 \%$. Our experience is based in a single country with a predominantly rural population, therefore distance and transportation costs maybe over-represented. Despite these limitations, the data presented have a number of strengths: large sample size, broad specialty mix and thorough demographic and barrier assessments all demonstrate how changing from a centralised to decentralised selection strategy can improve access to care for the poorest patients and those who experience multiple barriers to care.

\section{CONCLUSION AND RECOMMENDATIONS}

Barriers to surgical care are multifactorial and involve complex geographical and socioeconomic factors. 
NGOs and surgical providers in LMICs cannot simply offer free surgery and expect to reach those in need. Our data show that country-specific patient selection strategies can be developed to overcome principle barriers and allow patients in greatest need to access surgical care. Therefore, we make the following recommendations to NGOs and other stakeholders involved $\mathrm{n}$ the provision of surgical care in LMICs:

1. consider case-finding by travelling to patient's rural locations, rather than expecting the patient to travel to access care

2. design decentralised active case-finding strategies to reach the poorest communities, in collaboration with the community and its religious leaders

3. routine collection of demographic, wealth and prior barriers to care data (see online appendix file) can help determine patient profiles to assess if stakeholders are achieving their aims of improving access to surgical care to those who need it.

\section{Author affiliations}

${ }^{1}$ Department of Anaesthesia, Great Ormond Street Hospital, London, UK

${ }^{2}$ Hospital Department, Mercy Ships, Cotonou, Benin

${ }^{3}$ Hospital Department, Mercy Ships, Toamasina, Madagascar

${ }^{4}$ Department of Intensive Care, University Medical Center Utrecht, Utrecht, The Netherlands

${ }^{5}$ Department of Emergency Care, Lady Cilento Children's Hospital, South Brisbane, Queensland, Australia

${ }^{6}$ Department of Global Health and Social Medicine, Program in Global Surgery and Social Change, Harvard Medical School, Boston, Massachusetts, USA

${ }^{7}$ Department of Otolaryngology, Massachusetts Eye and Ear Infirmary, Boston, Massachusetts, USA

Contributors MCW, MH, JB, KR and MGS conceived and designed the study. All authors contributed to data acquisition. MCW and MGS contributed to data interpretation and analysis. MCW wrote the first draft of the manuscript, and all authors were involved in critical revision of the article and approved the final version for publication.

Funding Mark Shrime receives funds from the Damon Runyon Cancer Research Foundation and from the GE Safe Surgery 2020 project.

Competing interests None declared.

Ethics approval Mercy Ships Institutional Review Board.

Provenance and peer review Not commissioned; externally peer reviewed.

Data sharing statement There are no further unpublished data.

Open Access This is an Open Access article distributed in accordance with the Creative Commons Attribution Non Commercial (CC BY-NC 4.0) license, which permits others to distribute, remix, adapt, build upon this work non-commercially, and license their derivative works on different terms, provided the original work is properly cited and the use is non-commercial. See: http://creativecommons.org/ licenses/by-nc/4.0/

(C) Article author(s) (or their employer(s) unless otherwise stated in the text of the article) 2017. All rights reserved. No commercial use is permitted unless otherwise expressly granted.

\section{REFERENCES}

1. Alkire BC, Raykar NP, Shrime MG, et al. Global access to surgical care: a modelling study. Lancet Glob Health 2015;3:e316-23.
2. Weiser TG, Haynes AB, Molina G, et al. Estimate of the global volume of surgery in 2012: an assessment supporting improved health outcomes. Lancet 2015;385(Suppl 2):S11.

3. Shrime MG, Dare AJ, Alkire BC, et al. Catastrophic expenditure to pay for surgery worldwide: a modelling study. Lancet Glob Health 2015;3(Suppl 2):S38-S44.

4. Shrime MG, Dare A, Alkire BC, et al. A global country-level comparison of the financial burden of surgery. Br J Surg 2016;103:1453-61.

5. Massenburg BB, Jenny HE, Saluja S, et al. Barriers to Cleft Lip and Palate Repair Around the World. J Craniofac Surg 2016;27:1741-5.

6. Al-Shammari KF, Al-Ansari JM, Al-Khabbaz AK, et al. Barriers to seeking preventive dental care by Kuwaiti adults. Med Princ Pract 2007;16:413-9.

7. Gilbert CE, Lepvrier-Chomette N. Gender inequalities in surgery for bilateral cataract among children in low-income countries: a systematic review. Ophthalmology 2016;123:1245-51.

8. Irfan FB, Irfan BB, Spiegel DA. Barriers to accessing surgical care in Pakistan: healthcare barrier model and quantitative systematic review. J Surg Res 2012;176:84-94.

9. Lerman BJ, Alsan M, Chia NJ, et al. Beyond infrastructure: understanding why patients decline surgery in the developing world: an observational study in Cameroon. Ann Surg 2016.

10. Swanson JW, Yao CA, Auslander A, et al. Patient Barriers to Accessing Surgical Cleft Care in Vietnam: A Multi-site, CrossSectional Outcomes Study. World J Surg 2017;41:1435-46.

11. Ajibode $\mathrm{H}$, Jagun $\mathrm{O}$, Bodunde $\mathrm{O}$, et al. Assessment of barriers to surgical ophthalmic care in South-Western Nigeria. J West Afr Coll Surg 2012;2:38-50.

12. Boeck MA, Nagarajan N, Gupta S, et al. Assessing access to surgical care in Nepal via a cross-sectional, countrywide survey. Surgery 2016;160:501-8.

13. Kadaluru UG, Kempraj VM, Muddaiah P. Utilization of oral health care services among adults attending community outreach programs. Indian J Dent Res 2012;23:841-2.

14. Lin BM, White M, Glover A, et al. Barriers to surgical care and health outcomes: a prospective study on the relation between wealth, sex, and postoperative complications in the Republic of Congo. World $J$ Surg 2017;41:14-23-23.

15. Nagarajan N, Gupta S, Shresthra S, et al. Unmet surgical needs in children: a household survey in Nepal. Pediatr Surg Int 2015;31:389-95.

16. van Loenhout JA, Delbiso TD, Gupta S, et al. Barriers to surgical care in Nepal. BMC Health Serv Res 2017;17:72.

17. Zhang XJ, Jhanji V, Leung CK, et al. Barriers for poor cataract surgery uptake among patients with operable cataract in a program of outreach screening and low-cost surgery in rural China. Ophthalmic Epidemiol 2014;21:153-60.

18. Bolkan HA, Von Schreeb J, Samai MM, et al. Met and unmet needs for surgery in Sierra Leone: A comprehensive, retrospective, countrywide survey from all health care facilities performing operations in 2012. Surgery 2015;157:992-1001.

19. Bruno E, White MC, Baxter LS, et al. An evaluation of preparedness, delivery and impact of surgical and anesthesia care in Madagascar: a framework for a National surgical plan. World J Surg 2017;41:1218-24.

20. Baxter LS, Ravelojaona VA, Rakotoarison HN, et al. An Observational Assessment of Anesthesia Capacity in Madagascar as a Prerequisite to the Development of a National Surgical Plan. Anesth Analg 2017;124:2001-7.

21. Demographic Health Survey Program. Secondary Demographic Health Survey Program. http://dhsprogram.com.

22. Wilhelm TJ, Dzimbiri K, Sembereka V, et al. Task-shifting of orthopaedic surgery to non-physician clinicians in Malawi: effective and safe? Trop Doct 2017:49475517717178.

23. Shrime MG, Sekidde S, Linden A, et al. Sustainable Development in Surgery: The Health, Poverty, and Equity Impacts of Charitable Surgery in Uganda. PLoS One 2016;11:e0168867.

24. Shrime MG, Verguet S, Johansson KA, et al. Task-sharing or public finance for the expansion of surgical access in rural Ethiopia: an extended cost-effectiveness analysis. Health Policy Plan 2016;31:706-16.

25. Nguyen K, Bhattacharya SD, Maloney MJ, et al. Self-reported barriers to pediatric surgical care in Guatemala. Am Surg 2013;79:885-8. 